\title{
A novel nomogram to predict the overall survival in esthesinoeroblastoma
}

\author{
Lijie Jiang ${ }^{1 \dagger}$, Tengjiao Lin ${ }^{2 \dagger}$, Yu Zhang ${ }^{2}$, Wenxiang Gao ${ }^{1}$, Jie Deng ${ }^{1}$, Zhaofeng Xu', Xin Luo ${ }^{1}$, Zhaoqi Huang', \\ Fenghong Chen ${ }^{1}$, Jianbo Shi ${ }^{1}$ and Yinyan Lai ${ }^{1 *}$
}

\begin{abstract}
Background: Increasing evidence indicates that the pathology and the modified Kadish system have some influence on the prognosis of esthesioneuroblastoma (ENB). However, an accurate system to combine pathology with a modified Kadish system has not been established.

Methods: This study aimed to set up and evaluate a model to predict overall survival (OS) accurately in ENB, including clinical characteristics, treatment and pathological variables. We screened the information of patients with ENB between January 1, 1976, and December 30, 2016 from the National Cancer Institute Surveillance, Epidemiology, and End Results (SEER) program as a training cohort. The validation cohort consisted of patients with ENB at Sun Yat-sen University Cancer Center and The First Affiliated Hospital of Sun Yat-sen University in the same period, and 87 patients were included. The Pearson's chi-squared test was used to assess significance of clinicopathological and demographic characteristics. We used the Cox proportional hazards model to examine univariate and multivariate analyses. The model coefficients were used to calculate the Hazard ratios (HR) with 95\% confidence intervals $(\mathrm{Cl})$. Prognostic factors with a $p$-value $<0.05$ in multivariate analysis were included in the nomogram. The concordance index (c-index) and calibration curve were used to evaluate the predictive power of the nomogram.
\end{abstract}

Results: The c-index of training cohort and validation cohort are $0.737(95 \% \mathrm{Cl}, 0.709$ to 0.765$)$ and $0.791(95 \% \mathrm{Cl}$, 0.767 to 0.815$)$ respectively. The calibration curves revealed a good agreement between the nomogram prediction and actual observation regarding the probability of 3-year and 5-year survival. We used a nomogram to calculate the 3-year and 5-year growth probability and stratified patients into three risk groups.

Conclusions: The nomogram provided the risk group information and identified mortality risk and can serve as a reference for designing a reasonable follow-up plan.

Keywords: Esthesioneuroblastoma, Prognosis, Nomogram, Survival

\section{Background}

ENB is a rare sinonasal tumor, which derived from the olfactory epithelium at the top of nasal cavity and is also named olfactory neuroblastoma [1]. It was reported that

\footnotetext{
* Correspondence: Laiyy3@mail.sysu.edu.cn

${ }^{\dagger}$ Lijie Jiang and Tengjiao Lin contributed equally to this work.

'The Otorhinolaryngology Hospital, First Affiliated Hospital of Sun Yat-sen University, No.58 Zhongshan Er Road, Guangzhou, Guangzhou 510080, P.R. China

Full list of author information is available at the end of the article
}

approximately $6 \%$ of nasal cavity and paranasal sinus tumors were ENB [2-4]. Berger and his colleagues first described the malignant neoplasm in 1924, and ENB is known to show variable progression [5]. Dulguerov reported that the diagnosis difficulty, varying biological activity and lack of valid staging system with consensus may contribute to the variable progression in 2001 [6].

The Hyams grading system based on histological features was first described by Hyams [7]. Dulguerov also

(c) The Author(s). 2020 Open Access This article is licensed under a Creative Commons Attribution 4.0 International License, which permits use, sharing, adaptation, distribution and reproduction in any medium or format, as long as you give appropriate credit to the original author(s) and the source, provide a link to the Creative Commons licence, and indicate if changes were made. The images or other third party material in this article are included in the article's Creative Commons licence, unless indicated otherwise in a credit line to the material. If material is not included in the article's Creative Commons licence and your intended use is not permitted by statutory regulation or exceeds the permitted use, you will need to obtain permission directly from the copyright holder. To view a copy of this licence, visit http://creativecommons.org/licenses/by/4.0/ The Creative Commons Public Domain Dedication waiver (http://creativecommons.org/publicdomain/zero/1.0/) applies to the data made available in this article, unless otherwise stated in a credit line to the data. 
noted the possible role of histopathologic grading in predicting prognosis by meta-analysis [6]. In 2014, Saade reported that necrosis and mitosis were significant predictors of OS and progression-free survival (DFS) but not as individual parameters [8].

The staging classification of clinical data was first proposed by Kadish and his coworkers, which included three categories for ENB due to the shortcoming of the staging system [9]. Morita et al. modified the Kadish system by including group $\mathrm{D}$, which includes patients with metastasis to the cervical lymph nodes or distant site [10]. In 2001, Dulguerov proposed the modified Kadish staging system, which is more similar to the TMN tumor system, and the criteria were based on magnetic resonance imaging (MRI) and computed tomography (CT). The modified Kadish system for ENB is more reliable in assessing the anatomical sites of disease [6].

Jethanamest and Nalavenkata respectively reported the modified Kadish staging system as risk factor used to predict the prognosis of patients with ENB and guide tumor management and treatment $[11,12]$. Recently, the histopathology of the Hyams Grading System has been proven to affect the prognosis of ENBs and the treatment [13-17]. However, the single clinical staging system has not been shown to be an adequate predictor of outcome.

Our study aims to establish a nomogram based on clinical characteristics, treatment and pathological variables in predicting OS among patients with ENB. Finding a more suitable indicator for the prediction of ENB prognosis is critical.

\section{Methods}

\section{Patient data collection}

For the training cohort, histological feature code 9522 was used to identify all patients diagnosed with ENB from January 1, 1976, to December 30, 2016, in the SEER database. We signed and adhered to the data use agreement for SEER radiation therapy and chemotherapy information to obtain the chemotherapy and radiation therapy data from SEER database. Specific site codes C30.0, C31.0, C31.1, C31.2, C31.3, C31.8, C31.9 were used to identify the specific location of the tumor in the nasal cavity or paranasal. Although modified Kadish staging was not available in the SEER database, we used SEER extent of disease, collaborative stage extension, historic stage, and primary site to deduce modified Kadish staging. Jethanamest et al. and Tajudeen et al. used this method of modified Kadish stage derivation for SEER studies pertaining to ENB $[11,18]$. Extent of disease and collaborative staging extent codes for anatomic involvement of primary tumors were grouped and correlated with the appropriate modified Kadish stage as follows: confined to the nasal cavity (stage A), extension to the paranasal sinuses (stage B), extension beyond the nasal cavity and sinuses, including the cribriform plate and base of skull (stage C), and lymph node and distant metastases (stage D). We invited two experienced clinicians to derive the modified Kadish stage for the SEER cohort. When there are disagreements, they determined through consultation. We defined tumor differentiation grades I and II as low-grade tumors, and defined grades III and IV as high-grade tumors. The retrospective study followed the Helsinki Declaration (1964) and its later amendments or comparable ethical standards. No consent was required for the deidentified data, and no additional ethical approval processes were required for access to the database. Patient information was acquired by SEER"Stat software (version 8.3.6).

The inclusion criteria for the training cohort was as follows: 1. ENB with positive histological confirmation and not from an autopsy or death certificate; 2. active follow-up patients; and 3. known survival months after diagnosis and cause of death. The exclusion criteria for both the training cohort and the validation cohort were as follows: 1. unknown demographic information (age, race, sex); 2. unknown clinicopathological information (tumor grade and modified Kadish stage); 3. ENB was not the primary tumor if there were 2 or more; and 4 . the follow-up time was less than 1 month. A total of 639 patients were excluded due to unknow demographic and clinicopathological information, or not first tumor, or follow up time less than 1 month after treatment. The flow diagram of training cohort data selection is shown in supplementary Figure 1. After applying the screen criteria, 225 patients were included in the final SEER cohort.

For the validation cohort, ENB patient data were collected from Sun Yat-sen University Cancer Center and The First Affiliated Hospital of Sun Yat-sen University in the same period. This study was approved by the institutional review boards of the First Affiliated Hospital, Sun Yat-sen University, Guangzhou, China ([2020]111). All inclusion criteria were identical to those used in the SEER cohort. A total of 96 patients with pathologicproven ENBs were screened in this period, but 2 patients were excluded due to not first tumor and 7 patients were excluded because unknow demographic and clinicopathological information. After applying the screen criteria, 87 patients were included in the validation cohort. The Pearson's chi-squared test was used to assess significance of clinicopathological and demographic characteristics.

Modified Kadish classification and Hyams grading system The modified Kadish classification was used to classify primary tumor extension: stage $\mathrm{A}$ is confined to nasal cavity, stage $\mathrm{B}$ extends into paranasal sinuses, stage $\mathrm{C}$ 
extends beyond the paranasal sinuses and stage D presents cervical lymph node involvement (supplementary Table 1). For training cohort, tumor grade based on record of the SEER data base, while for the validation cohort, the tumor grade criterion was the Hyams Grading system (supplementary Table 2) and reviewed by two trained pathologists.

\section{Definition of OS and survival analysis}

OS was defined as the time from ENB diagnosis to the time of death or last follow-up. The OS length was calculated from the time of death for any cause or censoring. In this study, we used Kaplan-Meier analysis to calculate 3- and 5-year survival for covariates and used the log-rank test to determine statistical significance.

\section{Nomogram development}

Based on the results of multivariate analysis, a nomogram model was formulated. All variables with significant differences at $p<0.05$ in univariate analysis were included in the multivariate analysis. We used the Cox proportional hazards model for multivariate analyses. We used model coefficients to determine hazard ratios. Prognostic factors with a $p$-value $<0.05$ in multivariate analysis were included in the nomogram. The optimal cut-off values of age group and the nomogram score for risk group stratification were calculated by X-tile 3.6.1 software (Yale University, New Haven, CT, USA).

\section{Nomogram validation}

The nomogram's predictive power was evaluated by the c-index for both of training cohort and validation cohort. We used the c-index to evaluate the predictive power of the nomogram for both cohorts. The c-index was used to quantify the difference between the prediction and the actual situation [19]. Values ranged from 0.5 (no discrimination) to 1.0 (complete discrimination). A larger C-index predicts a more accurate prediction of the prognosis. The agreement between predicted survival and the observed survival after bias correction was quantified by calibration curves of the nomogram for the 3year and 5-year OS. Statistical analysis was conducted by $\mathrm{R}$ software version 3.5.2 ( $\mathrm{R}$ Foundation for Statistical Computing, Vienna, Austria; www.R-project.org). All calculated $p$ values were two-sided, and $p<0.05$ was considered statistically significant.

\section{Results}

\section{Clinicopathologic characteristics of patients and survival}

The training cohort comprised 225 patients with ENB who were recruited between 1976 and 2016. The nomogram was based on the training cohort, and the median OS time was 48 months (1-155 months). In the training cohort, $122(54.2 \%)$ patients were diagnosed at the age of 54 or younger. A total of $133(59.1 \%)$ patients were males, and $92(40.9 \%)$ were females. Additionally, 129 patients had a low-risk tumor grade, accounting for $57.3 \%$ of the total, and 96 (or $42.7 \%$ ) had a high-risk tumor grade. A total of $60.9 \%$ of the patients were diagnosed with stage $\mathrm{C}$ disease. Most of the patients in training cohort had received surgery (90.2\%) and radiotherapy treatment (68.0\%), while most of the patients had no chemotherapy treatment or had no information about chemotherapy (64.9\%). In terms of treatment options, due to the limitation of the SEER database, the sequence of chemotherapy with surgery and radiotherapy was unknown.

For the validation cohort, we studied 87 consecutive patients in the same period, and the median OS time was 29 months (1-208 months). Fifty-eight (66.7\%) patients were males and 29 (33.3\%) patients were females in the validation cohort. The most common age at diagnosis of these patients with ENB was $\leq 54$ (70.1\%). With regard to tumor stage, modified Kadish C stage (42.5\%) was most frequent, followed by B stage (29.9\%), D stage (26.4\%) and A stage (1.1\%). The majority of patients received radiotherapy (66.7\%). Only $41.4 \%$ of patients in the Chinese cohort had received surgical treatment (Table 1).

Patient characteristics and tumor characteristics were assessed by Kaplan-Meier survival analysis. The modified Kadish A or B group had the highest 5-year OS rate (89.4\%). The $\mathrm{C}$ group and $\mathrm{D}$ group represented 72.1 and $50.7 \%$, respectively (Fig. 1a). In terms of the tumor differentiation grade characteristics, patients in the highgrade group had a comparatively lower OS rate, reaching $63.9 \%$, while in the low-grade group, the 5-year OS rate was $81.8 \%$ (Fig. 1b). The 5 -year OS rates for training cohort patients younger than 55 years old, 55-69 years old and older than 70 were 83.1, 71.2 and $44.2 \%$, respectively (Fig. 1c). The 5-year OS of the low-risk group was $93.0 \%$, followed by the medium-risk group $(63.4 \%)$ and the high-risk group (28.3\%) (Fig. 1d).

\section{Independent prognostic factors of OS}

Univariate analyses have demonstrated that modified Kadish stage, gender, tumor differentiation grade, age at diagnosis, chemotherapy, and surgery are associated with OS. We included all of the above prognostic factors with $p<0.05$ in the multivariate analysis, and multivariate analysis showed that modified Kadish stage, age at diagnosis, and tumor differentiation grade were independent risk factors for patients with ENB. The detailed results of the multivariate analysis are presented in Table 2.

\section{Nomogram construction and risk stratification}

In the Cox model, modified Kadish stage, tumor differentiation grade, and age at diagnosis were independent 
Table 1 Demographic and clinicopathological characteristics of patients with ENB

\begin{tabular}{|c|c|c|c|}
\hline Characteristics & Training cohort $(N=225)(\%)$ & Validation cohort $(N=87)(\%)$ & $P$ value \\
\hline Gender (\%) & & & 0.136 \\
\hline Female & $92(40.9)$ & $29(33.3)$ & \\
\hline Male & $133(59.1)$ & $58(66.7)$ & \\
\hline Age (\%) & & & 0.034 \\
\hline$<=54$ & $122(54.2)$ & $61(70.1)$ & \\
\hline $55-69$ & $73(32.4)$ & $20(23.0)$ & \\
\hline$>=70$ & $30(13.3)$ & $6(6.9)$ & \\
\hline Tumor Grade (\%) & & & 0.304 \\
\hline Low & $129(57.3)$ & $56(64.4)$ & \\
\hline High & $96(42.7)$ & $31(35.6)$ & \\
\hline Modified Kadish(\%) & & & 0.002 \\
\hline A & $49(21.8)$ & $1(1.1)$ & \\
\hline B & $13(5.8)$ & $26(29.9)$ & \\
\hline C & $137(60.9)$ & $37(42.5)$ & \\
\hline D & $26(11.6)$ & $23(26.4)$ & \\
\hline Chemotherapy(\%) & & & 0.005 \\
\hline $\mathrm{N}$ & $146(64.9)$ & $42(48.3)$ & \\
\hline Y & $79(35.1)$ & $45(51.7)$ & \\
\hline Radiotherapy(\%) & & & 0.461 \\
\hline N & $72(32.0)$ & $29(33.3)$ & \\
\hline Y & $153(68.0)$ & $58(66.7)$ & \\
\hline Surgery(\%) & & & $<0.001$ \\
\hline N & $22(9.8)$ & $51(58.6)$ & \\
\hline Y & $203(90.2)$ & $36(41.4)$ & \\
\hline
\end{tabular}

$N$ No/Unknown, $Y$ Yes

prognostic factors revealed by multivariate analyses. Modified Kadish stage, tumor differentiation grade, and age at diagnosis were used to develop the nomogram for estimating 3- and 5-year OS (Fig. 2). To use a nomogram, lines are drawn to score the prognostic variables on the top point scale for an individual patient. The number of points received for each variable value and the score for each prognostic variable on the point scale are added together. The sum of scores is on the total point axis, and one line is drawn to the survival axis to convert to a 3- or 5-years probability.

Patients were subdivided into a low-risk group $(0 \leq$ score $\leq 57.5$ ), an intermediate-risk group (scoring $57.5<$ score $<157.5)$ and a high-risk group $(157.5 \leq$ score $\leq$ $300)$.

\section{Nomogram validation}

In this study, we performed both internal and external validation of the nomogram. The plotted calibration curves corresponded to the ideal plot (the $45^{\circ}$ line), which revealed a favorable agreement on the nomogram estimation and actual observation regarding the probability of 3-year and 5-year survival (Fig. 3a, b, c, d). In the training cohort, the model showed a high accuracy with a c-index of 0.737 (95\% CI, 0.709 to 0.765) which was higher than the modified Kadish staging system, at $0.614(95 \% \mathrm{CI}, 0.579$ to 0.649$)$. In the validation cohort, the nomogram prediction was 0.791 (95\% CI, 0.767 to 0.815 ) was also higher than for the modified Kadish staging system prediction $(0.674,95 \%$ CI, $0.643-$ 0.705 ). These results suggest that the nomogram was reasonably accurate, repeatable and had a better accuracy in predicting OS than the modified Kadish staging system.

\section{Discussion}

Recently, several disease centers have published their own treatment experience, but each single-center study of the disease was generally limited by the sample size, which had a certain impact on the accuracy of the results. Modified Kadish staging system was the most widely used ENB staging system [10, 20]. Recent studies have shown that the survival of patients with ENB was significantly associated with pathologic grade and age 


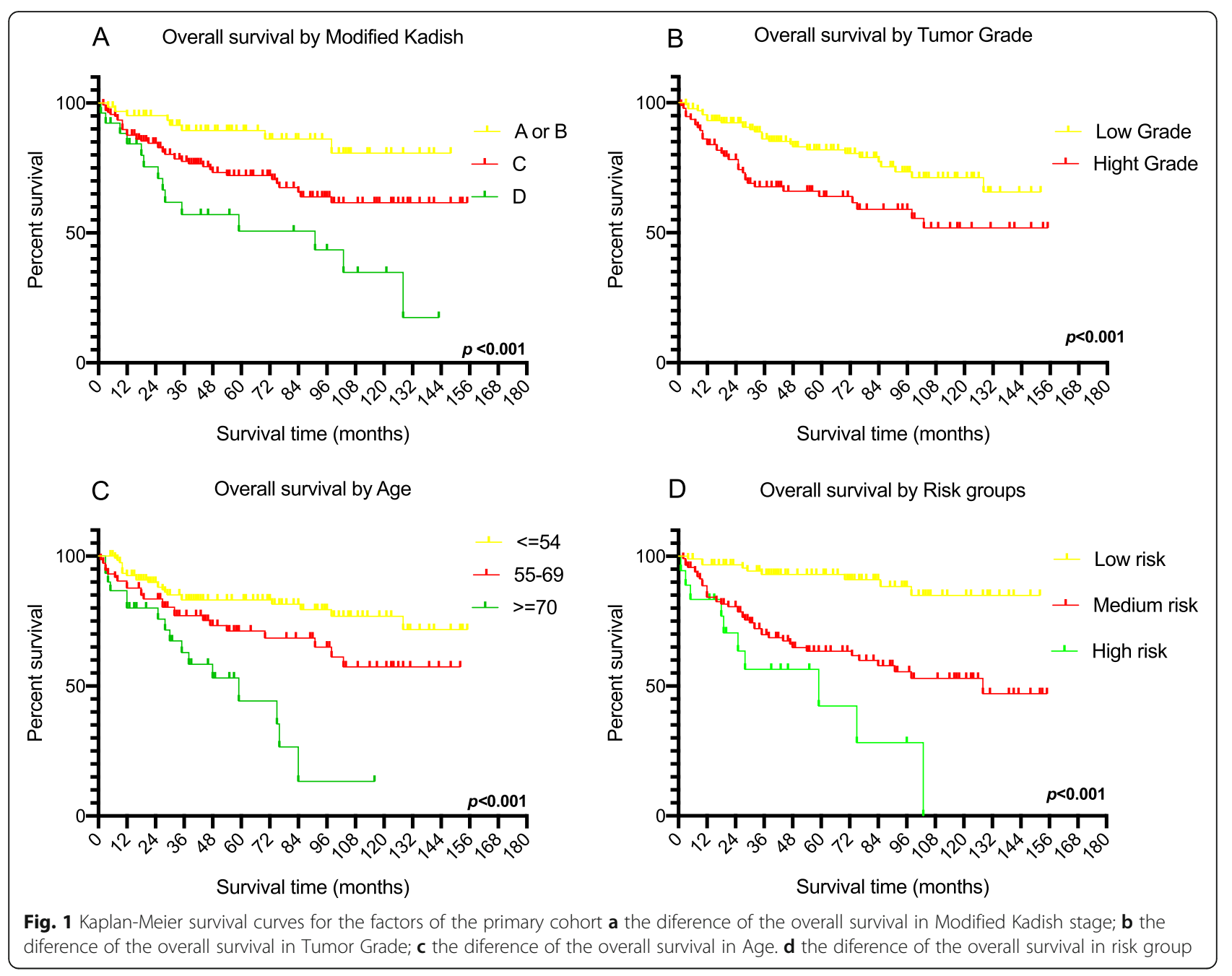

$[18,21]$. To the best of our knowledge, this is the first study to use the nomogram model to combine age, pathologic grade, and modified Kadish staging systems to predict the prognosis of patients with ENB.

Although surgery and chemotherapy were associated with patient outcomes in univariate analysis, they were not prognostic independent predictors in multivariate analysis. Patients receiving chemotherapy often have large local tumors or distant metastases [6]. For patients with locally advanced tumors, chemotherapy decreased the risks of systemic failure by acting on systemic micrometastasis [22]. For patients with distant metastases who did not undergo radical surgery, chemotherapy may be a suitable treatment and control the lesions. Compared with the SEER cohort, the Chinese cohort had relatively fewer patients undergoing surgery and a higher proportion of patients receiving chemotherapy. One reason for this phenomenon was that the proportion of distant metastasis in the Chinese cohort was significantly higher than that in the Western cohort $(26.4 \%$ vs. 11.6$)$. This may be due to bias caused by too small a sample size. Another potential reason was that Sun Yat-sen University Cancer Center and The First Affiliated Hospital of Sun Yat-sen University were two famous hospitals in China, a bias toward more advanced disease among those referred to these two hospitals. Last but not least, the lack of ascertainment in SEER and the inevitable selection bias might be weighted towards a surgical group.

The role of age in ENB is still controversial and unclear. In this study, the best cutoff values of 54 and 70 were calculated by X-tile [23], and the prognosis was the best in the group of patients younger than 54 years old. Although these patients all received the same treatment strategy, this study still showed different survival trends in three groups. Yin et al. showed that patients older than 60 years of age had a worse prognosis [21]. Previous studies have shown that young patients with ENB have more aggressive disease, but these patients are sensitive to chemotherapy and can achieve good results through a combination of chemotherapy and radiotherapy [24]. 
Table 2 Cox Proportional Hazards Regression Analyses of overall survival for ENB patients in the training Cohort

\begin{tabular}{|c|c|c|c|c|}
\hline \multirow[t]{2}{*}{ Variable } & \multicolumn{2}{|l|}{ Univariate analysis } & \multicolumn{2}{|c|}{ Multivariate analysis } \\
\hline & HR (95\%) & $P$ & HR (95\%) & $P$ \\
\hline Sex (\%) & Ref & & & \\
\hline Female & $1.392(0.826-2.344)$ & 0.214 & $\mathrm{NI}$ & \\
\hline Male & & & $\mathrm{NI}$ & \\
\hline \multicolumn{5}{|l|}{ Age(\%) } \\
\hline$<=54$ & Ref & & Ref & \\
\hline $55-69$ & $1.787(1.002-3.186)$ & 0.049 & $1.726(0.962-3.097)$ & 0.067 \\
\hline$>=70$ & $4.131(2.160-7.900)$ & $<0.001$ & 3.773 (1.950-7.505) & $<0.001$ \\
\hline \multicolumn{5}{|c|}{ Tumor Grade(\%) } \\
\hline Low & Ref & & Ref & \\
\hline High & $2.240(1.354-3.707)$ & $<0.001$ & $1.991(1.151-3.444)$ & 0.014 \\
\hline \multicolumn{5}{|c|}{ Modified Kadish } \\
\hline$A$ or $B$ & Ref & & Ref & \\
\hline$C$ & $2.536(1.187-5.42)$ & 0.016 & $1.950(0.892-4.263)$ & 0.094 \\
\hline $\mathrm{D}$ & $5.246(2.199-12.51)$ & $<0.001$ & $2.797(1.057-7.401)$ & 0.038 \\
\hline \multicolumn{5}{|c|}{ Chemotherapy } \\
\hline $\mathrm{N}$ & Ref & & Ref & \\
\hline Y & $1.857(1.126-3.061)$ & 0.015 & $1.161(0.635-2.122)$ & 0.628 \\
\hline \multicolumn{5}{|c|}{ Radiotherapy } \\
\hline $\mathrm{N}$ & Ref & & $\mathrm{NI}$ & \\
\hline Y & $0.725(0.435-1.209)$ & 0.218 & $\mathrm{Nl}$ & \\
\hline \multicolumn{5}{|l|}{ Surgery } \\
\hline $\mathrm{N}$ & Ref & & Ref & \\
\hline Y & $0.407(0.217-0.764)$ & 0.005 & $0.729(0.362-1.470)$ & 0.378 \\
\hline
\end{tabular}

$N$ No/Unknown, $Y$ Yes, NI Not include, Ref Reference, HR Hazard Ratio

We recommend that young, locally advanced patients enter Multi-Disciplinary Therapy Meeting (MDT) to discuss and determine treatment options.

The standard for pathological grading of ENB is the Hyams standard. At present, some studies have reported that pathological graded survival was significantly correlated and was an independent predictor of survival in patients with ENB [11, 25-27]. For Chinese cohort, the tumor differentiation criterion based on the Hyams Grading System while the SEER cohort used the tumor differentiation grading scheme. The indicators for evaluating cell differentiation included mitotic index and nuclear polymorphism, which were also part of the Hyams scoring system. Limited by the SEER database, it could not provide Hyams grading information, but the impact on nomogram might be slight. Tajudeen et al. considered that tumor differentiation grading scheme roughly corresponded to the Hyams grading scale [11]. Significant differences in survival can be seen in the pathological graded polarization of ENB, and high-grade pathological differentiation grades tend to have a worse prognosis [26]. In this study, we defined grade I and grade II tumors as low-grade tumors and defined grade III and grade IV tumors as high-grade tumors. In these two groups of patients, we observed significant differences in both training cohort and validation cohort, while in multivariate analysis, high-grade tumors were risk factors for prognosis. For SEER grading scheme may not be interpreted as a true Hyams grade, but it roughly corresponds to the Hymas grading scale. The bias caused by this method requires a large sample size cohort containing Hyams grading information as training cohort to reconstruct a nomogram. However, due to the rarity of ENB, the SEER database was the largest cohort that could be obtained, and variability was minimized by grouping patients into low-grade and high-grade tumor groups.

A number of studies evaluated the predictive power of the modified Kadish staging system [6, 20, 28]. Although it was partly confirmed that the modified Kadish staging system can effectively predict the prognosis of patients, some of them did not confirm its predictive efficacy. The reason for this phenomenon was the lack of sample size, selection bias, or a defect in the modified Kadish staging system itself. In the present study, we did not find statistically significant differences in survival between modified Kadish A and B, either in the SEER cohort or in the Chinese cohort. This was consistent with the conclusions of some previous studies [28]. Therefore, to improve statistical performance, stage $A$ and stage $B$ ENB patients were combined together. The prognosis of these patients was significantly better than that of patients with stage $\mathrm{C}$ and stage $\mathrm{D}$ disease.

The prognostic significance of clinical staging and pathologic grading were perhaps confounded often by each other $[6,25]$. These factors explained the limitations of using pathological grading and clinical grading alone to some extent. The use of the modified Kadish staging system and pathological grading system was not sufficiently accurate. We established a nomogram to predict the prognosis of patients. Based on the Cox regression risk model, the model calculated the likelihood of 3-year and 5-year survival based on the patient's age, clinical stage and tumor pathologic grade. Clinical application was simple and convenient. Here, we demonstrated a nomogram application example based on the calculation of the nomogram. The patient was a 55 -yearold male who was diagnosed with clinical stage $C$ in 2012. The tumor pathologic differentiation grade was a low-risk group (grade I well differentiated), and the patient underwent both surgery and radiotherapy. According to the nomogram, the measured probability of 3-year survival was about $83 \%$, and the 5 -year survival was about $80 \%$. This patient was in the low-risk group. When this new method of evaluating prognosis is 


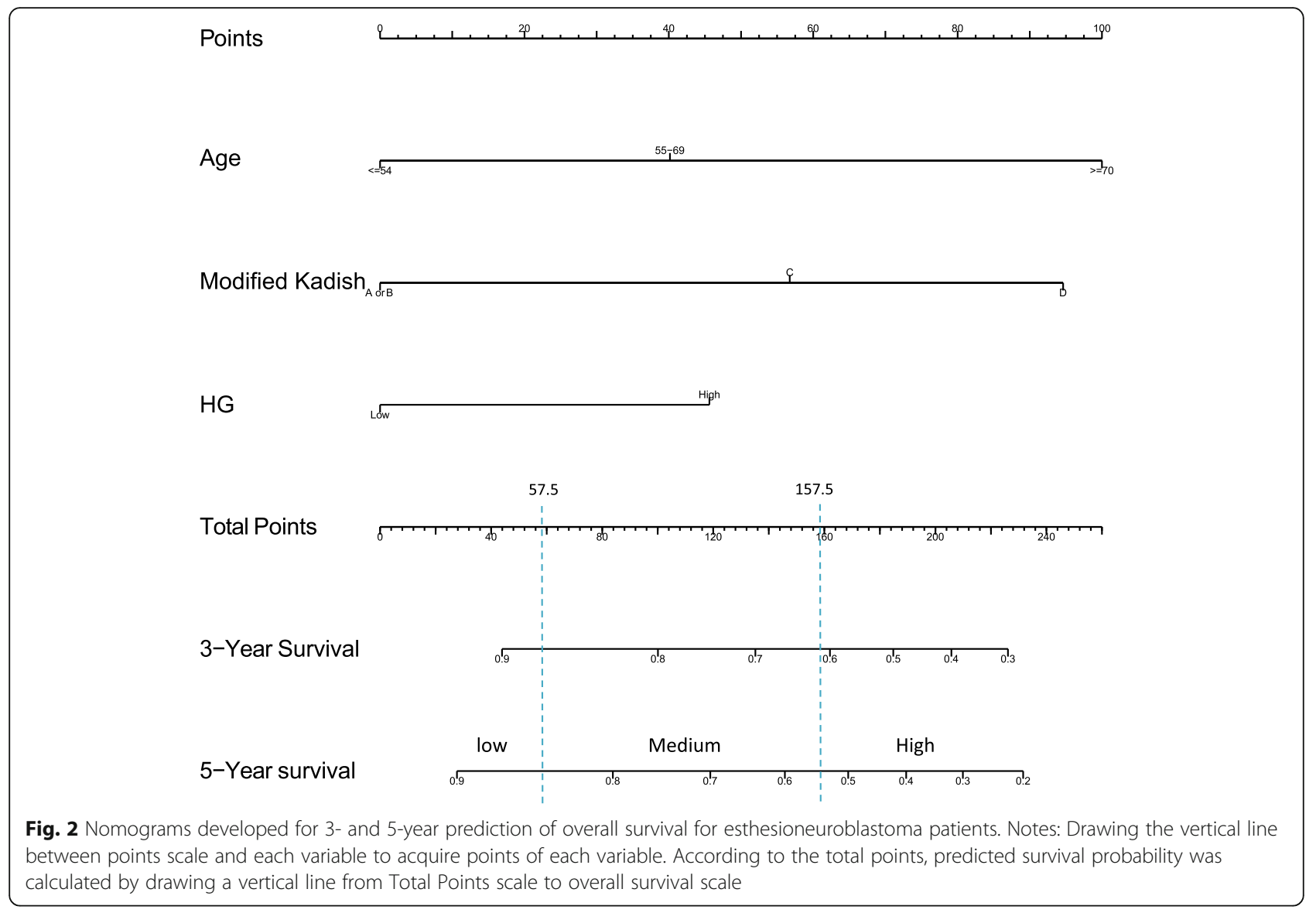

extended to patients in the non-SEER cohort, we recommended using this nomogram after the validation step. It can reduce the bias caused by selection and regional differences in medical levels. It may be necessary to build a nomogram with the data of the non-SEER population, and then determine the cut-off value of the risk stratification according to the actual situation of the population by using our methods.

The c-indexes for internal and external validation were 0.737 ( $95 \% \mathrm{CI}, 0.709$ to 0.765 ) and 0.791 (95\% CI, 0.767 to 0.815$)$, respectively, which showed that the present nomogram was a repeatable and accurate prognostic tool for predicting 3- and 5-year OS in patients. However, some of ENB patients could have a long naturel history, OS may not be the most relevant endpoint. Quality of life for ENB patients was one of dominant components of the treatment evaluation. Thus, DFS may be more relevant than OS, but SEER database only provides OS and disease-specific survival as primary endpoints. Further improvement of our nomogram by using patient series with data for quality of life and DFS is needed. Nonetheless, this nomogram could act as a tool to select high-risk patients and make individualized treatment and followup schedules.
There were several limitations in our study. First, this was a retrospective analysis study that inevitably had a selective bias. One of the enrollment criteria used positive histology only, which resulted in some patients who did not receive surgery and lacked pathological data were excluded from the cohort. A total of 599 patients were excluded due to unknow demographic and clinicopathological information and 279 out of 599 patients (46.6\%) had no surgery performed. The proportion of excluded patients who received surgery was lower than the proportion of enrolled patients (53.4\% vs. 90.2\%). This might increase the proportion of patients in the SEER cohort who received surgery. Second, the SEER database did not provide detailed chemotherapy information. In this study, we were unable to confirm information about the course of treatment for patients with chemotherapy, which may lead to bias in the treatment results. In addition, the detailed radiation therapy data are not provided, and it was hard to evaluate the treatment impact on the SEER cohort's patients. Third, the SEER database did not provide the patient's surgical methods, so it was impossible to make comparisons on the influence of the surgical approach. Finally, the SEER database did not provide patients' information about modified Kadish stage and Hyams grade. Modified 


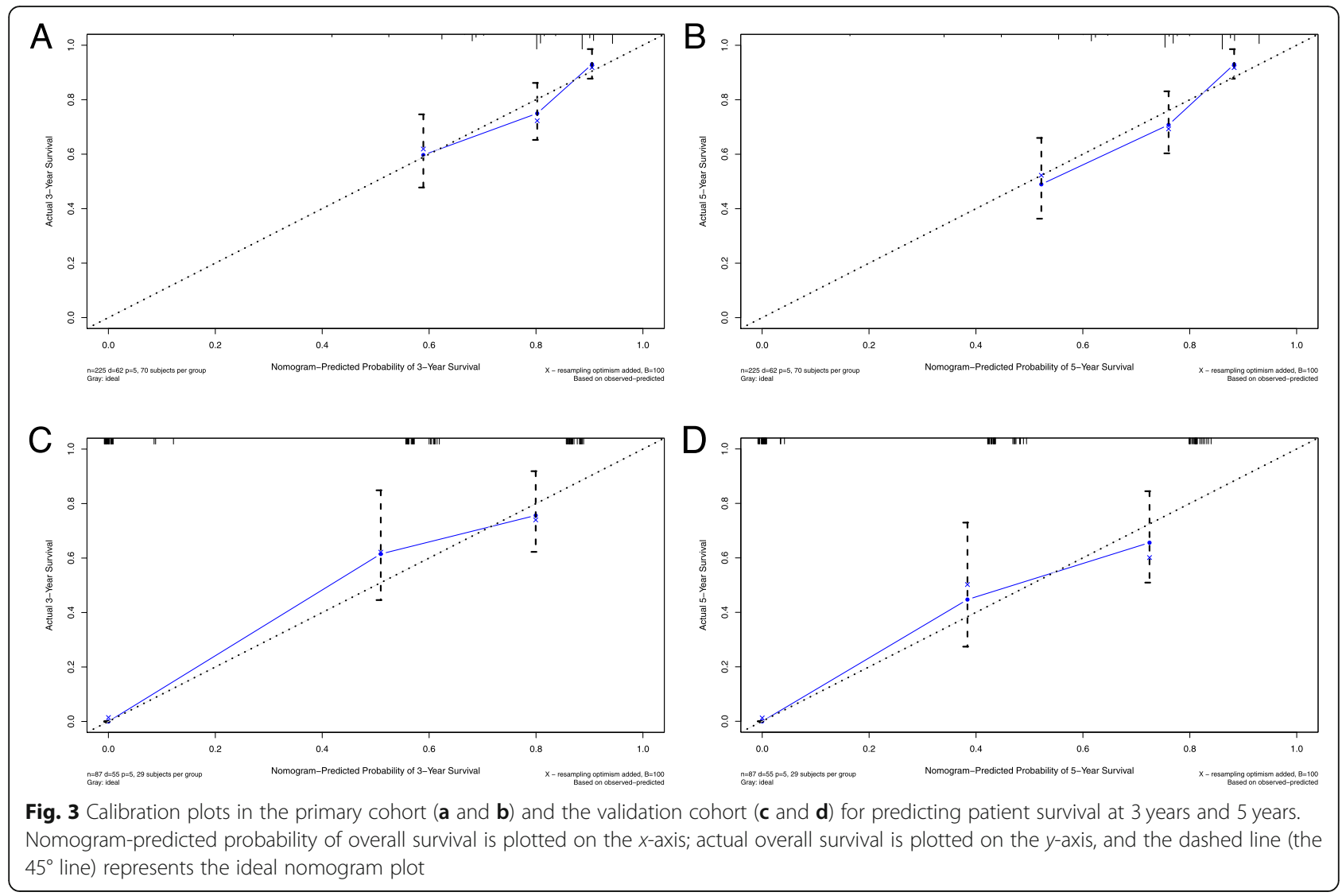

Kadish staging transformation depended on the accurateness of SEER data and its coding system. Nonetheless, the results were still novel, we successful provided insight into the utility of the nomogram and to verify the repeatability and practicability of the nomogram in validation cohort.

\section{Conclusion}

The study explored a nomogram based on clinical characteristics, treatment and pathological variables in predicting OS among patients with ENB. The present study identified modified Kadish staging system, tumor differentiation grade, and age at diagnosis as independent prognostic variables for the OS rates of patients with ENB. We used a nomogram to calculate the 3-year and 5-year growth probability and stratified patients into three risk groups. The nomogram provided the risk group information and identified mortality risk and can serve as a reference for a more reasonable follow-up plan.

\section{Supplementary information}

Supplementary information accompanies this paper at https://doi.org/10. 1186/s12885-020-07435-7.

Additional file 1: Figure S1. Flowchart of included population in this study.

\section{Additional file 2}

\section{Abbreviations}

ENB: Esthesioneuroblastoma; OS: Overall Survival; SEER: Surveillance, Epidemiology, and End Results; HR: Hazard ratios; Cl: Confidence interval; Cindex: Concordance index; DFS: Progression-free survival; MRI: Magnetic resonance imaging; CT: Computed tomography; MDT: Multi-Disciplinary Therapy Meeting

\section{Acknowledgements}

Not applicable.

\section{Authors' contributions}

$L J$ and TJL contributed to most of collecting data, data analysis and drafting the manuscript; YZ, WXG and LX contributed to data acquisition; ZFX and ZQH contributed to the statistical analysis; FHC and JD contributed to interpretation of data; JBS and YYL contributed to the study design, revision of the manuscript. Joint first or senior authorship: LJJ and TJL should be considered joint first author. All authors have read and approved the final version of the manuscript.

\section{Funding}

This study was supported by National Natural Science Foundation of China (81300814) in collection, analysis, and interpretation of data as well as in writing the manuscript. This study was also supported by Natural Science Foundation of China (81470069) in the design of the study and collection, analysis, and interpretation of data. The findings and conclusions in this manuscript are those of the authors and do not necessarily represent the views of Natural Science Foundation of China.

\section{Availability of data and materials}

The datasets used and/or analysed during the current study are available from the corresponding author on reasonable request. 


\section{Ethics approval and consent to participate}

The retrospective study protocol was reviewed and approved by the Ethics Committee of The First Affiliated Hospital of Sun Yat-sen University, Guangzhou, China ([2020]111), with waiver of informed consent for Chinese cohort. Informed patient consent was not required for data released by the SEER database.

The publication of research details under the Creative Commons Attribution License 4.0.

\section{Consent for publication}

Not applicable.

\section{Competing interests}

No conflict of interest exists in the submission of this manuscript. No conflict of interest exists in financial.

\section{Author details}

1The Otorhinolaryngology Hospital, First Affiliated Hospital of Sun Yat-sen University, No.58 Zhongshan Er Road, Guangzhou, Guangzhou 510080, P.R. China. ${ }^{2}$ Sun Yat-sen University Cancer Center, Guangzhou, P.R. China.

\section{Received: 17 April 2020 Accepted: 17 September 2020}

Published online: 14 October 2020

\section{References}

1. Takahashi H, Ohara S, Yamada M, Ikuta F, Tanimura K, Honda Y. Esthesioneuroepithelioma: a tumor of true olfactory epithelium origin. Acta Neuropathol. 1987;75(2):147.

2. Bradley PJ, Jones NS, Robertson I. Diagnosis and management of esthesioneuroblastoma. Curr Opin Otolaryngol Head Neck Surg. 2003;11(2): $112-8$.

3. Lund VJ, Howard D, Wei W, Spittle M. Olfactory neuroblastoma: past, present, and future? Laryngoscope. 2003;113(3):502.

4. Song CM, Won TB, Lee CH, Kim DY, Rhee CS. Treatment modalities and outcomes of olfactory neuroblastoma. Laryngoscope. 2012;122(11):2389-95.

5. Berger L, Luc R, Richard D. L'esthesioneuroepitheliome olfactif. Bull Assoc Fr Etude Cancer. 1924;13:410-21.

6. Dulguerov P, Allal AS, Calcaterra TC. Esthesioneuroblastoma: a meta-analysis and review. Lancet Oncol. 2001;2(11):683-90.

7. Hyams VJ. Olfactory neuroblastoma. In: Hyams VJ, Baksakis JG, Michaels L, editors. Tumors of the upper respiratory tract and ear. Washington DC: Armed Forces Institute of Pathology; 1988. p. 240-8.

8. Bell D, Saade R, Roberts D, Ow TJ, Kupferman M, Demonte F, Hanna EY. Prognostic utility of Hyams histological grading and Kadish-Morita staging Systems for Esthesioneuroblastoma Outcomes. Head Neck Pathol. 2014;9(1): 51-9.

9. Kadish S, Goodman M, Wang CC. Olfactory neuroblastoma. A clinical analysis of 17 cases. Cancer. 1976:37:1571-6.

10. Morita A, Ebersold MJ, Olsen KD, Foote RL, Lewis JE, Quast LMJN. Esthesioneuroblastoma: prognosis and management. Neurosurgery. 1993; 32(5):706-14.

11. Jethanamest D, Morris LG, Sikora AG, Kutler DI. Esthesioneuroblastoma: a population-based analysis of survival and prognostic factors. Arch Otolaryngol Head Neck Surg. 2007;133(3):276-80.

12. Nalavenkata SB, Sacks R, Adappa ND, Palmer JN, Purkey MT, Feldman MD, Schlosser RJ, Snyderman CH, Wang EW, Woodworth BA. Olfactory neuroblastoma: fate of the neck--a long-term multicenter retrospective study. Otolaryngol Head Neck Surgery. 2016;154(2):383.

13. Malouf GG, Casiraghi O, Deutsch E, Guigay J, Temam S, Bourhis J. Low- and high-grade esthesioneuroblastomas display a distinct natural history and outcome. Eur J Cancer. 2013:49(6):1324-34.

14. Kaur G, Kane AJ, Sughrue ME, Madden M, Oh MC, Sun MZ, Safaee M, ElSayed I, Aghi M, Mcdermott MW. The prognostic implications of Hyam's subtype for patients with Kadish stage C esthesioneuroblastoma. J Clin Neurosci. 2013;20(2):281.

15. Meyer C, Hamersley ER, Manosalva RE, Torske K, Mcintyre N, Mitchell A. Olfactory neuroblastoma with divergent differentiation: an unusual histologic finding in a rare tumor. Head Neck Pathol. 2017;11(4):531.

16. Saade RE, Hanna EY, Bell D. Prognosis and biology in esthesioneuroblastoma: the emerging role of Hyams grading system. Curr Oncol Rep. 2015;17(1):423.
17. Xiong L, Zeng XL, Guo CK, Liu AW, Huang L. Optimal treatment and prognostic factors for esthesioneuroblastoma: retrospective analysis of 187 Chinese patients. BMC Cancer. 2017;17(1):254.

18. Tajudeen BA, Arshi A, Suh JD, St John M, Wang MB. Importance of tumor grade in Esthesioneuroblastoma survival: a population-based analysis. JAMA Otolaryngol Head Neck Surg. 2014;140(12):1124

19. lasonos A, Schrag D, Raj GV, Panageas KS. How to build and interpret a Nomogram for Cancer prognosis. J Clin Oncol. 2008;26(8):1364-70.

20. Kumar R. Esthesioneuroblastoma: multimodal management and review of literature. World J Clin Cases. 2015;3(9):774.

21. Yin Z, Wang Y, Wu Y, Zhang X, Wang F, Wang P, Tao Z, Yuan Z. Age distribution and age-related outcomes of olfactory neuroblastoma: a population-based analysis. Cancer Manag Res. 2018;10:1359-64.

22. Porter AB, Bernold DM, Giannini C, Foote RL, Link MJ, Olsen KD, Moynihan TJ, Buckner JC. Retrospective review of adjuvant chemotherapy for esthesioneuroblastoma. J Neuro-Oncol. 2008;90(2):201-4.

23. Camp RL, Dolled-Filhart M, Rimm DL. X-tile: a new bio- informatics tool for biomarker assessment and outcome- based cut-point optimization. Clin Cancer Res. 2004;10:7252-9.

24. Bisogno G, Soloni P, Conte M, Podda M, Ferrari A, Garaventa A, Luksch R, Cecchetto $\mathrm{G}$. Esthesioneuroblastoma in pediatric and adolescent age. A report from the TREP project in cooperation with the Italian Neuroblastoma and Soft Tissue Sarcoma Committees. BMC Cancer. 2012;12(1):117.

25. Kane AJ, Sughrue ME, Rutkowski MJ, Aranda D, Mills SA, Buencamino R, Fang S, Barani IJ, Parsa AT. Posttreatment prognosis of patients with esthesioneuroblastoma. J Neurosurg. 2010;113(2):340-51.

26. Van Gompel J, Giannini C, Olsen K, Moore E, Piccirilli M, Foote R, Buckner J, Link M. Long-term outcome of Esthesioneuroblastoma: Hyams grade predicts patient survival. J Neurol Surg B Skull Base. 2012;73(05):331-6.

27. Goshtasbi K, Abiri A, Abouzari M, Sahyouni R, Wang BY, Tajudeen BA, Hsu FPK, Cadena G, Kuan EC. Hyams grading as a predictor of metastasis and overall survival in esthesioneuroblastoma: a meta-analysis. Int Forum Allergy Rhinol. 2019;9(9):1054-62.

28. Joshi RR, Husain Q, Roman BR, Cracchiolo J, Yu Y, Tsai J, Kang J, McBride S, Lee NY, Morris L, et al. Comparing Kadish, TNM, and the modified Dulguerov staging systems for esthesioneuroblastoma. J Surg Oncol. 2019; 119(1):130-42.

\section{Publisher's Note}

Springer Nature remains neutral with regard to jurisdictional claims in published maps and institutional affiliations.

Ready to submit your research? Choose BMC and benefit from:

- fast, convenient online submission

- thorough peer review by experienced researchers in your field

- rapid publication on acceptance

- support for research data, including large and complex data types

- gold Open Access which fosters wider collaboration and increased citations

- maximum visibility for your research: over $100 \mathrm{M}$ website views per year

At $\mathrm{BMC}$, research is always in progress.

Learn more biomedcentral.com/submission 